\title{
Application of Web Mining Using Clonal Selection Algorithm
}

\author{
Y1lmaz Atay and Halife Kodaz
}

\begin{abstract}
The Clonal Selection Algorithm is one of the most important algorithms of Artificial Immune Systems. In addition, it is has taken place in multiple studies in recent years. In this study, most visited web pages have been identified from internet access file by using a Clonal Selection Algorithm. In here, Clonal Selection Algorithm is applied to web mining association analysis. Necessary changes according to the association rules have been made in selection and mutation mechanisms of the Clonal Selection Algorithm. Results showed that the proposed method can be applied successfully for association rules.
\end{abstract}

Index Terms-Artificial immune systems, association rules, clonal selection algorithm, web mining.

\section{INTRODUCTION}

Web mining is the detection and analysis of information needed from internet records and web files by using some data obtaining techniques. In this study, the Clonal Selection Algorithm (CSA) has been used in web mining. Necessary changes have been made in selection and mutation mechanisms of the CSA. These changes were made appropriate according to the web mining. Logical patterns obtained from web records and the most visited web pages were determined. The determination procedure was carried out as quickly as possible. Many studies about extraction of meaningful patterns from web data have been carried out up to now. These studies have subjected web content mining, web structure mining and web usage mining which are types of web mining. Artificial intelligence techniques are used quite a lot in this area. Some studies in the field of web mining are given below.

Many studies have been conducted so far on web mining. Some of these studies are as follow. Agrawal et al. have proposed and used the mathematical model of association rule [1]. Srivastava et al., in their studies indicated the basic concepts and general information about web usage mining. Intent of their study is to gather information about the difficulties encountered on web usage mining and provides recommendations for overcoming these challenges [2]. Uğuz et al., achieve meaningful information from user access records by applying Apriori algorithm on users' web data records in their studies [3]. Gao has stated that data mining about association rule occurs in two phases, finding all frequently passing items and production of strong association rules from these passing frequently items. Apriori algorithm

Manuscript received February 20, 2013; revised April 25, 2013. This work was supported in part by the Scientific Research Project of Selçuk University. Application of Web Mining Using Clonal Selection Algorithm.

The authors are with Computer Engineering Department, Engineering Faculty, Selçuk University, Konya-Turkey (e-mail: yilmazatay@selcuk.edu.tr, hkodaz@selcuk.edu.tr). which is used for the first phase of association rule is the most popular and classic algorithm used in detection of frequently passing items. In this algorithm, data and features are evaluated with boolean association rules [4]. Kuo et al., by using artificial neural networks and genetic algorithms have analyzed ways of web browser on the internet shopping. In their works, number of clicks on web pages by users and tracking of data path have been discussed. Then, meaningful information has been obtained by applying artificial neural networks and genetic algorithms on these data [5]. Tuğ et al. have found sequential visited web pages from internet records by genetic algorithm method in their works [6]. Gezer et al. made analysis of web mining and web pages. In their studies they examined web server log files by using two software named as WUMweb and WUMprep. So that they have identified the general characteristics of the web pages [7].

In the second section of this study, a description of web usage mining which is a type of web mining, association analysis, CSA and the proposed method have been given. In the third section the results of the application are given. Finally, assessments have been made about the study.

\section{MATERIALS AND METHODS}

\section{A. Web Usage Mining}

In this study, an important work has been done in the field of web usage mining which is one of web mining types. Also to subtract significant patterns from internet records CSA has been proposed. By applying CSA on records of web pages visited by users, $n$ number most visited web pages has been found as soon as possible. Where $n$ is a user-specified number of visited web pages. Fig. 1 presents the study fields related to web usage mining [8].

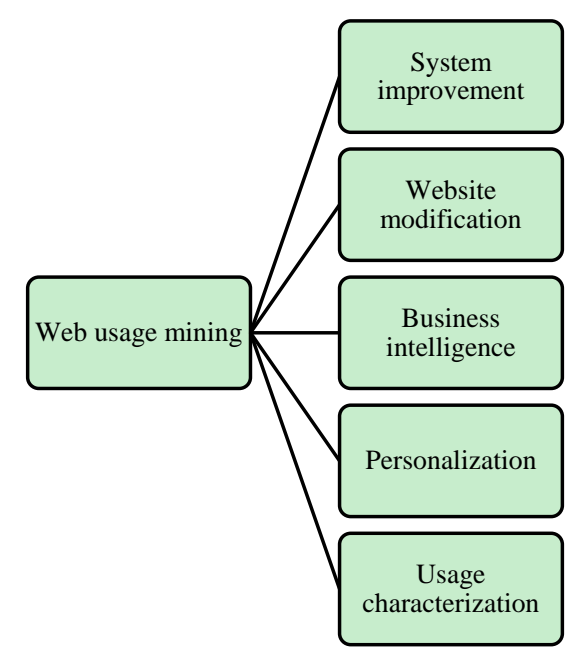

Fig. 1. Web usage mining working areas [8]. 
Web usage mining uses secondary data which is obtained from the interaction of internet users with the web while web content and web structure mining uses real data which is often described as the primary data in the web. Web usage data includes web server access logs, proxy server logs, browser records, user profiles, cookies, mouse clicks, page shifts and data such as the results of the interaction [9].

\section{B. Association Analysis}

Association rule is the analyzing of historical data and determination of association behavior within these data [10]. Association analysis is the extraction of meaningful association from complex data. Extraction of association rules for association analysis is performed by one of many statistical methods and artificial intelligence techniques. In this study, a method of association rules based on the CSA is proposed.

By applying association rules on web pages records, information can be gathered about the association of the pages visited during the same sessions. In this way, visited pages with proposed CSA can be obtained quickly. The presence of the most frequently visited web pages through the data has been achieved by necessary changes being made on the CSA. This process carried out quickly with the proposed method.

\section{Clonal Selection Algorithm}

CSA is model of the response of an immune system to an antigenic stimulus making use of natural immune system. When an antigen enters the body, sub-population of $\mathrm{B}$ lymphocyte cells derived from the bone marrow produces antibodies [11]. Antibodies stimulation to antigens, cloning the induced antibodies, destruction of unstimulated ones, selection and hypermutation process are in the CSA [11]. The fundamental basis of the CSA is the reproduction of the antibodies recognizing antigens. Block diagram of the CSA is given in Fig. 2. There are six steps in Fig. 2. These steps as follows [12]:

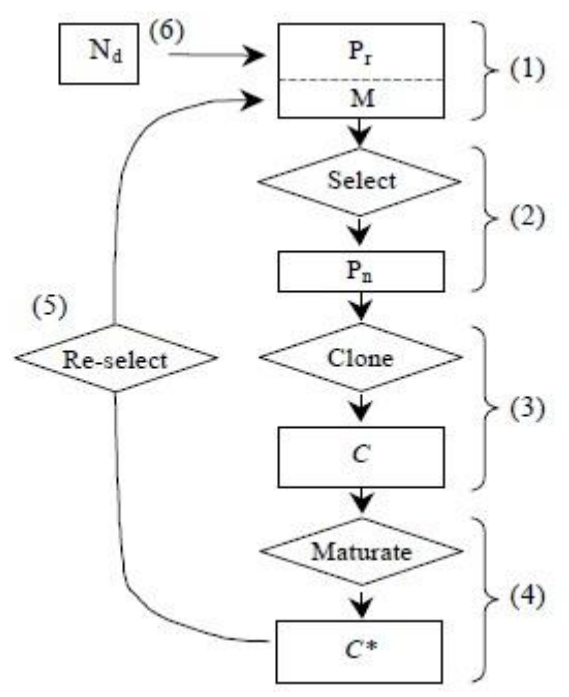

Fig. 2. Block diagram of the CSA [12].

Step 1: Generate a set $(P)$ of candidate solutions, composed of the subset of memory cells $(M)$ added to the remaining $\left(P_{r}\right)$ population $\left(P=P_{r}+M\right)$;

Step 2: Determine (Select) the $n$ best individuals of the population $\left(P_{n}\right)$, based on an affinity measure;
Step 3: Reproduce (Clone) these $n$ best individuals of the population, giving rise to a temporary population of clones $(C)$. The clone size is an increasing function of the affinity with the antigen;

Step 4: Submit the population of clones to a hypermutation scheme, where the hypermutation is proportional to the affinity of the antibody with the antigen. A maturated antibody population is generated $\left(C^{*}\right)$;

Step 5: Re-select the improved individuals from $C^{*}$ to compose the memory set $M$. Some members of $P$ can be replaced by other improved members of $C^{*}$;

Step 6: Replace $d$ antibodies by novel ones (diversity introduction). The lower affinity cells have higher probabilities of being replaced.

\section{The Proposed Method}

CSA is proposed for association analysis used within the web page for obtaining the logical data records. Every step of algorithm described in Section II part C is adapted in web association problem. All steps of this process are explained below.

Step 1: Defining the parameters: number of population, number of iterations, mutation rate, cloning rate, the number of association;

Step 2: Selecting a user-specified $n$ unit association;

Step 3: The creation of $\mathrm{m}$ dimensional antibody as the number of population;

Step 4: Transmission and assessment of the antibodies to the associated;

Step 5: Selection of "cloning rate $x$ number of population" antibody through the population;

Step 6: Create a value between $0-1$ and compare it to mutation rate, if this value is smaller than the rate of mutation defeat otherwise mutate;

Step 7: Repeat the above procedure for the set of all the clones;

Step 8: Find the best local association number;

Step 9: Compare the best local and global association number and update the best global;

Step 10: Terminate the iteration and save the best global antibody and association.

\section{EXPERIMENTAL RESULTS}

Association rule proposed for CSA is coded in Matlab. Web pages obtained from the records of a web page are numbered with an index. For example, "www.google.com.tr" web address is numbered with "20", "www.selcuk.edu.tr" web address is numbered with "10" and "www.isa-conf.org" web address is numbered with "1". These records were transferred to the file. Then, for association analysis proposed method was applied step by step as shown in Section II part D. Input panel of the implementation coded in Matlab and result panel which gives the best global results are provided respectively in Fig. 3 and Fig. 4.

In the program, as given in Fig. 3, the number of web page (association number) entered together is 3 , the number of population is 50 , the number of iterations is 100 , the cloning rate is 0.6 and mutation rate is 0.8 . Web pages records kept in the file were analyzed using the proposed method. The result obtained with the proposed method as soon as possible is "20-10-1" values (Xbest). These values are numbered in advance. The user most entered web page addresses are 
"www.google.com.tr", "www.selcuk.edu.tr" and "www.isa-conf.org" respectively. The number of detection of these records with proposed algorithm represents the number 7 which is the best value (Fbest number) in the global. The accuracy of Xbest and Fbest values have been checked and tested from the file.

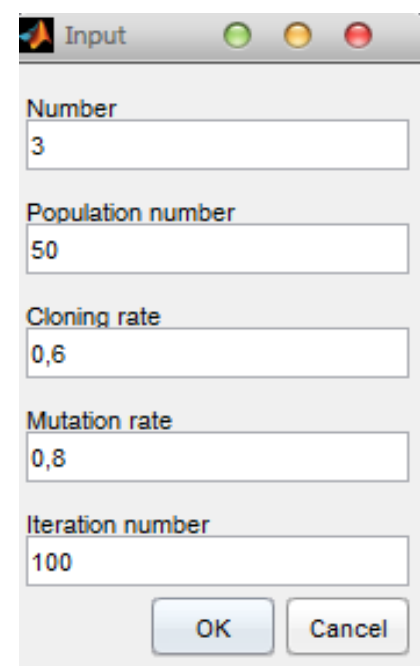

Fig. 3. Initial parameter values.

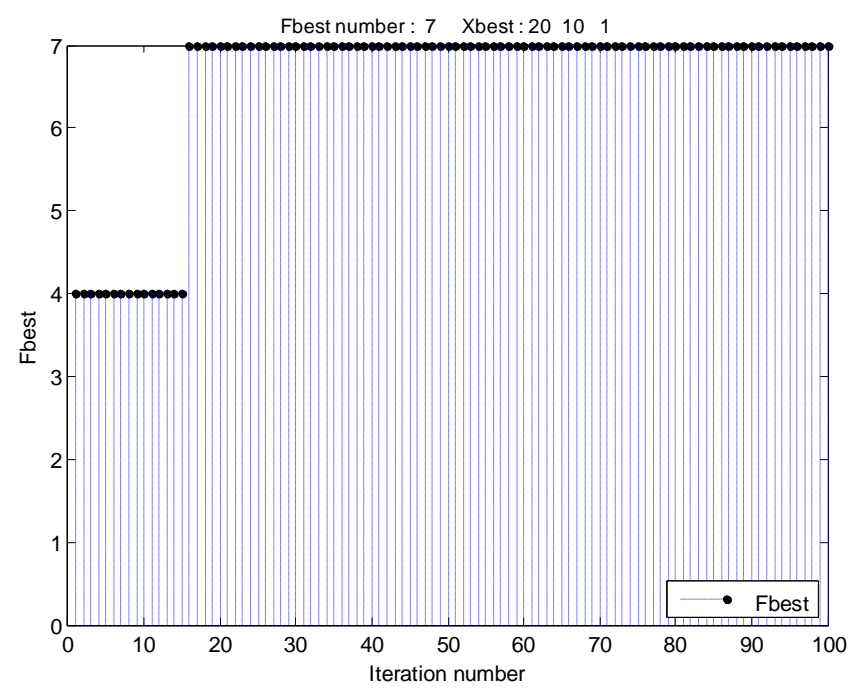

Fig. 4. The results of association rules.

\section{CONCLUSION}

In this paper, a study was conducted on association analysis which has an important place in web usage mining. In this study, for determination of association analysis of web pages records CSA has been applied. Finding the visited web pages quickly and successfully with the CSA has been achieved. This process tested on more complex data in a short time and found to be very useful, especially in terms of time. When the results are examined the implementation of CSA is not only been successful on association analysis but also it has been implemented on the most web mining successfully. Thus, meaningful patterns can be obtained quickly out of the website data that appears meaningless. However, with modifications deemed necessary on antibody selection and mutation mechanisms of CSA more successful results can be achieved.

\section{ACKNOWLEDGMENT}

This study has been supported by Scientific Research Project of Selçuk University.

\section{REFERENCES}

[1] R. Agrawal, T. Imielinski, and A. Swami, "Mining association rules between sets of items in large databases," in Proc. the ACM SIGMOD International Conference on Management of Data (ACMSIGMOD '93), Washington, USA, 1993, pp. 207-216.

[2] J. Srivastava, R. Cooley, M. Deshpande, and P. Tan, "Web usage mining: discovery and applications of usage patterns from web data," SIGKDD Exploartions, vol. 2, no. 1, pp. 1-12, 2000.

[1] H. Uğuz, H. Kodaz, E. Çomak, and Ö. K. Baykan, "Apriori Algoritması Kullanılarak Web Kullanım Madenciliği Yönteminin Web Log Kayitlarina Uygulanmas1," IJCI Proceeding of International Conference on Signal Processing, vol. 1, no. 2, ISSN 1304-2386, 2003.

[2] W. Gao, "A hierarchical document clustering algoritm," M.S. thesis, Dalhousie University, Halifax, Nova Scotia, 2004.

[3] R. J. Kuo, J. L. Lio, and C. Tu, Integration of art2 neural network and genetic k-means algorithm for analyzing web browsing paths in electronic commerce, Elsevier, 0167-9236 doi:10.1016/j.dss, 2004, pp. 355-374.

[4] E. Tuğ, A. M. Şakiroğlu, and A. Arslan, "Automatic discovery of the sequential accesses from web log data files via a genetic algorithm," Knowledge-Based Systems, vol. 19, pp. 180-186, 2006.

[5] M. Gezer, Ç. Erol, and S. Gülseçen, "Bir web sayfasının web madenciliği ile analizi," Akademik Bilisim 2007, Dumlupınar University, Kutahya, Turkey, 2007.

[6] B. Mobasher, R. Cooley, and J. Srivastava, "Automatic personalization based on web usage mining," Communications of the ACM, vol. 43, no. 8, pp. 142-151, 2000.

[7] B. Bulut, "Veri madenciligi yöntemlerinin incelenmesi ve uygulamaları," M.S. thesis, Applied Science Institution, Firat University, Turkey, 2006.

[8] W. J. Frawley, G. Piatetsky-Shapiro, and C. J. Matheus, "Knowledge Discovery Databases: An Overview, in Knowledge Discovery in Databases," AAAI 58 AI Magazine, Cambridge, pp. 1-27, 1991.

[9] L. N. De Castro and F. J. Von Zuben, "Immune and neural network models: theoretical and empirical comparisons," International Journal of Computational Intelligence and Applications, vol. 1, no. 3, pp. 239-257, 2001.

[10] L. N. De Castro and F. J. Von Zuben, "The clonal selection algorithm with engineering applications," in Workshop Proceedings of GECCO, 2000, pp. 36-37.

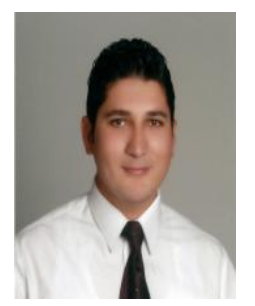

Yılmaz Atay was born in Adana on May 18, 1987 He graduated from Computer Engineering Department of Selçuk University with B.Sc. in 2010. After graduation, he worked in private sector for a short amount of time. He achieved His M. Sc. in Computer Engineering Department of the same University in 2012. Now he is Research Assistant of Computer Engineering at Selçuk University besides working on his Ph.D. degree. His research interests are Clonal Selection, Optimization, Artificial Intelligent Algorithm, Machine Vision and Embedded Systems.

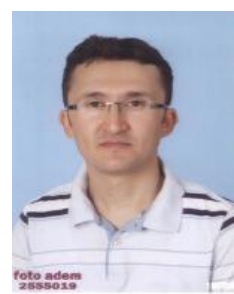

Halife Kodaz was born in Sivas on April 15, 1977. He graduated from Computer Engineering Department of Selçuk University with B.Sc. degree in 1999, from Computer Engineering Department of Selçuk University with M.Sc. degree in 2002 and from Electrical-Electronics Engineering Department of Selçuk University with Ph.D. degree in 2008. He works as an assistant professor at Selçuk University. His research interests are Artificial Immune Systems and Machine Learning Algorithms. 